\title{
Shifting paradigms in lung cancer
}

\author{
Vincent T DeVita, Jr
}

The Practice Point by Azzoli on an important article by Winton et al. ${ }^{1}$ highlights a landmark in the chemotherapy of cancer. Adjuvant chemotherapy significantly prolongs lung cancer survival, and is now indicated in patients with stage IB and II non-small-cell lung cancer. Four studies were required, ${ }^{2-5}$ capped by the Winton et al. article (which even warranted an editorial in the New York Times), to convince people of the survival benefits of adjuvant therapy. The absolute differences in the survival curves between the control and treated arms in the four studies cited range from $4 \%$ to $15 \%$, but the real differences (i.e. percentage improvement between the treated and control arm/survival of patients in the control arm) range from $13 \%$ to $27 \%$. So, this improvement is not only of biological interest but it is also clinically useful.

Adjuvant therapy is applicable in tumors that often present with locoregional disease, where the results of resection and/or radiotherapy are not sufficient because of the presence of micrometastases outside the area of presentation. The treatments used are not actually tested in this population but, instead, are developed in patients with advanced disease of similar histology, where they rarely produce lasting clinical benefit. These studies are often cited by critics as wasteful, expensive examples of the futility of treating advanced solid tumors. But the payoff, of course, occurs when marginally effective treatments in patients with advanced disease are applied as adjuvant therapy. This fact is often overlooked, creating a serious disconnect in the mind of the public over the value of chemotherapy. The common solid tumors-breast, colorectal and non-small-cell lung cancer-all have useful adjuvant therapies. All were developed in decades of tedious testing of single drugs and combinations of drugs in seemingly endless variations, in patients with metastatic disease. Mortality rates from breast and colorectal cancer are declining in the US, partly as a result of adjuvant treatment, and adjuvant treatments
We need to be more ingenious

in our testing

of these

potential new

combinations...

so we do

not have to

wait decades

for the next

paradigm shift.

VT DeVita, Jr is the Editor-in-Chief of Nature Clinical Practice Oncology.

Competing interests The author declared he has competing interests; go to the article online for details.

www.nature.com/clinicalpractice doi:10.1038/ncponc0345 for lung cancer should accelerate the decline that is already in motion as a result of smoking cessation, the preferred way to decrease mortality rates from lung cancer.

These solid tumors, unlike leukemias, lymphomas and testicular cancers, are also more drug-resistant at the outset, which accounts for the failure to cure any significant number of patients with advanced disease. Why this is so is still a mystery, but tumors such as lung and colorectal cancers, and to a lesser degree, breast cancers, arise after exposure to an external environment where they come into contact with hundreds or thousands of external toxins. Cells that become cancerous are not necessarily the ones damaged by the toxins, but rather the ones that survive the exposure and are all the wiser for the experience. We have a poor appreciation of this kind of resistance and yet it is a major roadblock to more successful treatment.

Here again we might look for another paradigm shift. Newer therapies, such as antibodies that target epidermal growth factor receptors and small-molecule kinase inhibitors, while not overly impressive by themselves, appear to reset the resistance mechanisms, and might prove most useful in conjunction with more standard cytotoxic chemotherapy. The dilemma is that regulatory agencies make it difficult to gain approval of these drugs because of their limited usefulness alone, despite good toxicity profiles compared with standard cytotoxic drugs. In fact, with a few notable exceptions, the more specific these agents are against a target, the less activity they have alone, which portends more regulatory hurdles. We need to be more ingenious in our testing of these potential new combinations, in patients with both advanced and locoregional disease, so we do not have to wait more decades for the next paradigm shift.

Supplementary information, in the form of a reference list, is available on the Nature Clinical Practice Oncology website. 